\title{
Synthesis and application of a novel star-hyperbranched poly(acrylic acid) for improved dental restoratives
}

\author{
Jun Zhao, Yiming Weng, Dong Xie*
}

Department of Biomedical Engineering, Purdue School of Engineering and Technology, Indiana University-Purdue University at Indianapolis, Indianapolis, USA.

Email: dxie@iupui.edu

Received 15 September 2010; revised 27 September 2010; accepted 30 September 2010.

\begin{abstract}
A new star-hyperbranched poly(acrylic acid) has been synthesized and incorporated into dental glassionomer cement for enhanced mechanical strengths. The effects of arm number and branching on viscosity of the polymer aqueous solution and mechanical strengths of the formed experimental cement were evaluated. It was found that the higher the arm number and the more the branching, the lower the viscosity of the polymer solution as well as the mechanical strengths of the formed cement. It was also found that the experimental cement exhibited significantly higher mechanical strengths than commercial Fuji II LC. The experimental cement was $51 \%$ in CS, $55 \%$ in compressive modulus, $118 \%$ in DTS, $82 \%$ in FS, $18 \%$ in FT and $85 \%$ in KHN higher than Fuji II LC. The experimental cement was only $6.7 \%$ of abrasive and $10 \%$ of attritional wear depths of Fuji II LC in each wear cycle. It appears that this novel experimental cement is a clinically attractive dental restorative and may potentially be used for high-wear and high-stress-bearing site restorations.
\end{abstract}

Keywords: Star-Hyperbranched Poly(Acrylic Acid); Light-Cured Glass-Ionomer Cement; Atom-Transfer Radical Polymerization; Mechanical Strength

\section{INTRODUCTION}

There are three major dental filling restoratives including dental amalgam, composite resins and glass-ionomer cements. Glass-ionomer cements are one of the most promising restoratives in dentistry [1]. Since their invention, these cements have been successfully applied in dentistry for almost 30 years [1-4]. The success of these cements is attributed to the facts that they are known for their unique properties such as direct adhesion to tooth structure and base metals $[5,6]$, anticariogenic properties due to release of fluoride [7], thermal compatibility with tooth enamel and dentin because of low coefficients of thermal expansion similar to that of tooth structure [8], minimized microleakage at the tooth-enamel interface due to low shrinkage [8], and low cytotoxicity $[9,10]$.

An acid-base reaction between calcium and/or aluminum cations released from a reactive glass and carboxyl anions pendent on polyacid describes the setting and adhesion mechanism of GICs [2,11]. Despite numerous advantages of GICs, brittleness, low tensile and flexural strengths have limited the current GICs for use only at certain low stress-bearing sites such as Class III and Class V cavities [1,2]. Much effort has been made to improve the mechanical strengths of GICs $[1,4,11]$ and the focus has been mainly on improvement of polymer backbone or matrix [1,4,11,12-18]. Briefly two main strategies have been applied. One is to incorporate hydrophobic pendent (meth)acrylate moieties onto the polyacid backbone in GIC to make it become light- or redox-initiated resin-modified GIC (RMGIC) [12-15,17] and the other is to directly increase molecular weight (MW) of the polyacid [16-18]. As a result, the former has shown significantly improved tensile and flexural strengths as well as handling properties [12-15,17]. The strategy of increasing MW of the polyacid by either introducing amino acid derivatives or $\mathrm{N}$-vinylpyrrolidone has also shown enhanced mechanical strengths [16-18]; however, the working properties were somehow decreased because strong chain entanglements formed in these high MW linear polyacids resulted in an increased solution viscosity $[16,17]$. It is known that viscosity is inversely proportional to MW of a polymer and a polymer with high MW often show both high mechanical strengths and viscosity $[7,8]$. So far, all the polyacids used in commercial GIC formulations have been linear polymers and using high MW of these linear polyacids has been limited due to the viscosity issue.

Polymers with star, hyperbranched or dendritic shapes often demonstrate low solution or melt viscosity because 
these molecular structures behave similar to a solution of hard spheres and exhibit limited chain entanglements, which is beneficial to polymer processing [19,20]. Recently, we have developed a light-curable glass-ionomer system composed of the 4-arm star polymer [21]. The polymer was synthesized via an advanced polymerization technique - atom-transfer radical polymerization (ATRP). The formed GIC system has no monomer in it. Because of this unique nature, the system has demonstrated substantially higher mechanical strengths as compared to Fuji II LC [21,22]. The main purpose of using star-shape polymer was to improve the mechanical strengths of the current GICs by altering the molecular architectures of the polymers. The strategy has been found valid [21,22].

This paper reports the synthesis and characterization of a new star-hyperbranched poly(acrylic acid) or poly (AA), use of the polymer to formulate the cements with glass fillers, and evaluation of the mechanical strengths of the formed cements.

\section{MATERIALS AND METHODS}

\subsection{Materials}

2-Hydroxylethylacrylate (HEA), 2-bromoisobutyryl bromide (BIBB), pentaerythritol, 1,1,1-tris-(hydroxymethyl)propane, dipentaerythritol, triethylamine (TEA), pyridine, $\mathrm{CuBr}, \mathrm{N}, \mathrm{N}, \mathrm{N}$ ',N",N"-pentamethyldiethylenetriamine (PMDETA), dl-camphoroquinone (CQ), 2-(dimethylamino) ethyl methacrylate (DMAEMA), tert-butyl acrylate (t-BA), glycidyl methacrylate (GM), hydrochloric acid (37\%), diethyl ether, dioxane, N,N-dimethylformamide (DMF) and tetrahydrofuran (THF) were used as received from VWR International Inc (Bristol, CT) without further purifications. Fuji II LC cement and Fuji II LC glass powders were used as received from GC America Inc (Alsip, IL).

\subsection{Synthesis of the 2-(2-Bromoisobutyryloxy) Ethyl Acrylate (BIEA) Initiator}

The initiator BIEA was synthesized as shown below: to a flask containing HEA (9.7 mmol), TEA (10.7 mmol) and THF (15 ml), a solution of BIBB (10.2 mmol) in THF (25 $\mathrm{ml})$ was added dropwise to keep the temperature below $5^{\circ} \mathrm{C}$ with the help of an ice-water bath. The reaction was run at room temperature for additional $4 \mathrm{~h}$ before the formed precipitates were filtered. The filtrate was then concentrated under a reduced pressure to afford a yellowish oil with a yield of $74 \%$. The synthesis scheme is shown in Figure 1.

\subsection{Synthesis of the 4-Arm Pentaerythritol Tetrakis (2-Bromoisobutyrate) Initiator}

The 4-arm initiator was synthesized following the pub- lished procedures [21]. Briefly, to a reactor charged with TEA (10 ml), pentaerythritol $(11.0 \mathrm{mmol})$ and THF (20 $\mathrm{ml})$, a solution of BIBB $(81.0 \mathrm{mmol})$ in THF $(25 \mathrm{ml})$ was added dropwise with stirring at room temperature. After addition was completed, additional $1 \mathrm{~h}$ was added to complete the reaction. The solution was washed with $5 \% \mathrm{NaOH}$ and $1 \% \mathrm{HCl}$, followed by extracting with ethyl acetate. The extract was dried with anhydrous $\mathrm{MgSO}_{4}$, concentrated in vacuo and crystallized. The final product was re-crystallized from diethyl ether. The 3-arm and 6-arm initiators were synthesized in a similar way as described above except that 1,1,1-tris-(hydroxymethyl)-propane and dipentaerythritol were used as a core instead.

\subsection{Synthesis of the GM-Tethered Star-Hyperbranched Poly(AA)}

The GM-tethered star-hyperbranched poly(AA) was synthesized via three steps: synthesis of star-hyperbranched poly(t-BA) via ATRP, conversion of poly(t-BA) to poly(AA) and tethering of GM onto poly(AA). For synthesis of poly(t-BA), to a flask containing dioxane, a mixture of BIEA, 4-arm initiator, PMDETA (ligand) and t-BA was added in a predetermined ratio. After the above solution was degassed and nitrogen-purged via three freeze-thaw cycles, $\mathrm{CuBr}$ (catalyst) was incorporated. The solution was then heated to $120^{\circ} \mathrm{C}$ to initiate the ATRP [23]. The proton nuclear magnetic resonance $\left({ }^{1} \mathrm{HNMR}\right)$ spectrometer was used to monitor the reaction. After the polymerization was complete, the poly(t-BA) polymer was precipitated from water. $\mathrm{CuBr}$ and PMDEMA were removed by re-precipitated from dioxane/water. For conversion of poly(t-BA) to poly(AA), the poly(t-BA) polymer was hydrolyzed in a mixture containing dioxane and $\mathrm{HCl}$ (37\%) under refluxed condition for $18 \mathrm{~h}$ [24]. The formed poly(AA) was dialyzed against water until the $\mathrm{pH}$ became neutral. The purified star-hyperbranched poly(AA) was obtained through freeze-drying. For GM tethering [21,22], to a flask containing the star-hyperbranched poly(AA) and DMF, a mixture of GM, DMF, and pyridine (1\% of GM, by weight) was added dropwise. Under a nitrogen blanket, the reaction was run at $60^{\circ} \mathrm{C}$ for $5 \mathrm{~h}$ and then kept at room temperature overnight. The polymer tethered with GM was recovered by precipitation from diethyl ether, followed by drying in a vacuum oven at room temperature. The overall synthesis scheme is shown in Figure 1.

\subsection{Characterization}

The synthesized initiator and polymers were characterized by ${ }^{1} \mathrm{HNMR}$ spectroscopy using a $500 \mathrm{MHz}$ Bruker NMR spectrometer (Bruker Avance II, Bruker BioSpin Corporation, Billerica, MA). The deuterated methyl sulfoxide 
<smiles>OCC(CO)(CO)CO</smiles>

(1)<smiles>CC(C)(Br)C(=O)OCC(COC(=O)C(C)(C)Br)(COC(=O)C(C)(C)Br)OC(=O)C(C)(C)Br</smiles>

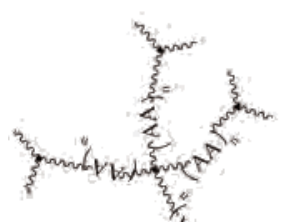<smiles>CC(C)CC(CC(C)C)C(CC(C)C)(CC(C)C)NC(C)C</smiles><smiles>CC(C)CC(C)CC(CC(C)C)CC(C)C</smiles>

(4)

(3)

4)<smiles>C=CC(=O)OCCOC(=O)C(C)(Br)Br</smiles>

(2)/<smiles>CCC(=O)OCCO</smiles><smiles>CCC(C)C</smiles><smiles>CCC(C)C(C)C</smiles>

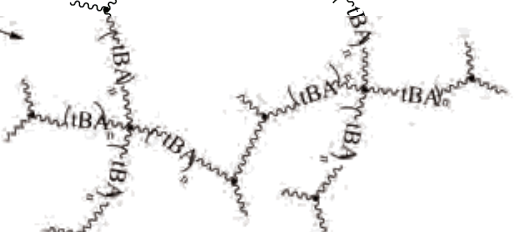

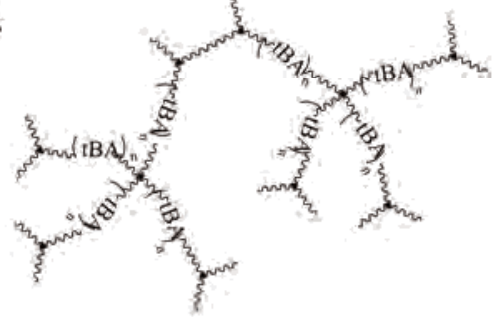

(5)

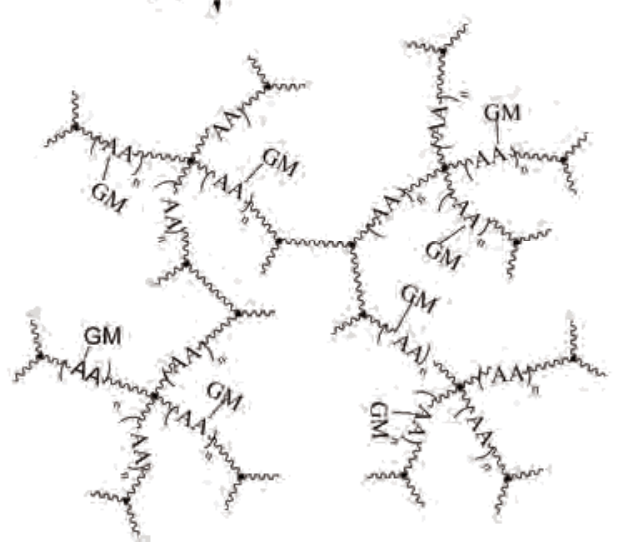

Figure 1. Schematic diagrams for preparation of the GM-tethered star-hyperbranched poly(AA): (1) synthesis of the 4-arm BIBB; (2) synthesis of BIEA initiators; (3) synthesis of poly(t-BA) via ATRP; (4) hydrolysis of poly(t-BA); (5) GM tethering.

(d-DMSO) and chloroform $\left(\mathrm{CDCl}_{3}\right)$ were used as solvents. The molecular weight (MW) and molecular weight distribution (MWD) of the synthesized poly (t-BA)s were determined in THF via a Waters GPC unit (Waters Corp., Milford, MA) with standard GPC techniques, using a polystyrene standard.
The viscosities of the liquids formulated with the GM-tethered star-hyperbranched poly(AA)s and distilled water were determined at $23^{\circ} \mathrm{C}$ using a programmable cone/plate viscometer (RVDV-II + CP, Brookfield Eng. Lab. Inc., Middleboro, MA).

The fracture and wear surfaces of the selected speci- 
mens from the FS, FT and wear tests were observed at a magnification of $1,500 \times$ using a scanning electron microscope (Model JSM 5310, JOEL Ltd, Tokyo, Japan). The specimens were vacuum sputter-coated with goldpalladium (Au-Pd), and a vacuum was used to dehydrate the coated specimens before SEM analysis.

\subsection{Sample Preparation}

The experimental cements were formulated with a twocomponent system (liquid and powder) [22]. The liquid was formulated with the GM-tethered polymer, water (polymer/water $(\mathrm{P} / \mathrm{W})$ ratio $=70 / 30$, by weight), CQ (photo-initiator, $0.9 \%$, by weight) and DMAEDA (activator, 1.8\%). Fuji II LC glass powder was used to formulate the experimental cements with a powder/liquid ratio of 2.7 unless specified. Fuji II LC cement was used as control and prepared per manufacturer's instruction where the $\mathrm{P} / \mathrm{L}$ ratio $=3.2$.

Specimens were fabricated at room temperature according to the published protocols $[21,22]$. Briefly, the specimens were prepared for different tests following the geometries below: 1) cylindrical specimens ( $4 \mathrm{~mm}$ in diameter $\times 8 \mathrm{~mm}$ in length) for compressive strength (CS); 2) disk specimens ( $4 \mathrm{~mm}$ in diameter $\times 2 \mathrm{~mm}$ in thickness) for diametral tensile strength (DTS); 3) rectangular specimens ( $3 \mathrm{~mm}$ in width $\times 3 \mathrm{~mm}$ in thickness $\times 25 \mathrm{~mm}$ in length) for flexural strength (FS); 4) rectangular specimens ( $4 \mathrm{~mm}$ in width $\times 2 \mathrm{~mm}$ in thickness $\times 20 \mathrm{~mm}$ in length), fitted with a sharp blade for generating 2-mmlong notch, for fracture toughness (FT) [25]; 5) disk specimens ( $4 \mathrm{~mm}$ in diameter $\times 2 \mathrm{~mm}$ in height), where the smooth surface at the diametral side was generated by pressing the cement against a microscopic slide before setting, for Knoop hardness; and 6) rectangular specimens ( $4 \mathrm{~mm}$ in width $\times 2 \mathrm{~mm}$ in thickness $\times 10 \mathrm{~mm}$ in length) for wear tests. All the specimens were exposed to blue light (EXAKT 520 Blue Light Polymerization Unit, GmbH, Germany) for 2 min, followed by conditioning at $37^{\circ} \mathrm{C}$ in $100 \%$ humidity for $15 \mathrm{~min}$ and then in distilled water for $24 \mathrm{~h}$ prior to testing.

\subsection{Evaluation}

CS, DTS, FS and FT tests were performed on a screwdriven mechanical tester (QTest QT/10, MTS Systems Corp., Eden Prairie, MN), with a crosshead speed of 1 $\mathrm{mm} / \mathrm{min}$. The FS and FT tests were performed in threepoint bending, with a span of $20 \mathrm{~mm}$ and $16 \mathrm{~mm}$, respectively, between supports. The sample sizes were $n=6-8$ for each test. CS was calculated using an equation of CS $=P / \pi r^{2}$, where $P=$ the load at fracture and $r=$ the radius of the cylinder. DTS was determined from the relationship DTS $=2 P / \pi d t$, where $P=$ the load at fracture, $d=$ the diameter of the cylinder and $t=$ the thickness of the cylinder. FS was obtained using the expression FS = $3 P \mathrm{l} / 2 b d^{2}$, where $P=$ the load at fracture, $l=$ the distance between the two supports, $\mathrm{b}=$ the breadth of the specimen, and $d=$ the depth of the specimen. FT was calculated from the equation $K_{I C}=\frac{P \cdot S}{B \cdot W} f(a / W)$, where $K_{I C}$ $=$ the index for FT, $P=$ the load at fracture, $S=$ the distance between supports, $a=$ the length of notch, $B=$ the thickness, and $W=$ the width of specimen. The $f$ is a function of $(a / W)$, as shown below [25]:

$$
f(x)=\frac{3 x^{0.5}\left[1.99-x(1-x)\left(2.15-3.93 x+2.7 x^{2}\right)\right]}{2(1+2 x)(1-x)^{1.5}}
$$

The hardness test was performed on a micro-hardness tester (LM-100, LECO Corporation, MI) using a diamond indenter with $25 \mathrm{~g}$ load and $30 \mathrm{~s}$ dwell time [26]. Knoop hardness number (KHN) was averaged from six readings for each sample.

The wear test was conducted using the Oregon Health Science University (OHSU) oral wear simulator (Prototech, Portland, OR) employing ceramic antagonists to produce both abrasive and attritional wear $[27,28]$. The test was performed following the published procedures [29] with a slight modification. Briefly, after polishing with sand paper, the specimen embedded in the mold was tightened into an individual wear chamber, followed by the addition of a food like slurry consisting of $1.0 \mathrm{~g}$ ground poppy seed, $0.5 \mathrm{~g}$ PMMA powder and $5 \mathrm{ml}$ distilled water. The abrasion force was set at $20 \mathrm{~N}$ and the attrition force at $90 \mathrm{~N}$. The specimen was subject to 70,000 wear cycles at a frequency of $1 \mathrm{~Hz}$. The worn specimen was analyzed using an optical surface profilometer (Surftronic 3+, Taylor Hobson Ltd, Leicester, England) [29]. Both abrasive and attritional wear depths were obtained per manufacturer's recommendation, averaging from three traces. Four specimens were tested to obtain a mean wear value for each material or formulation.

\subsection{Statistical Analysis}

One-way analysis of variance (ANOVA) with the post hoc Tukey-Kramer multiple-range test was used to determine significant differences of the measured properties among the materials in each group. A level of $\alpha=$ 0.05 was used for statistical significance.

\section{RESULTS}

\subsection{Synthesis and Characterization}

\subsubsection{Characterization of the Synthesized Initiators and Polymers}

Figure 2 shows the ${ }^{1} \mathrm{HNMR}$ spectra of t-BA, 4-arm BIBB, 


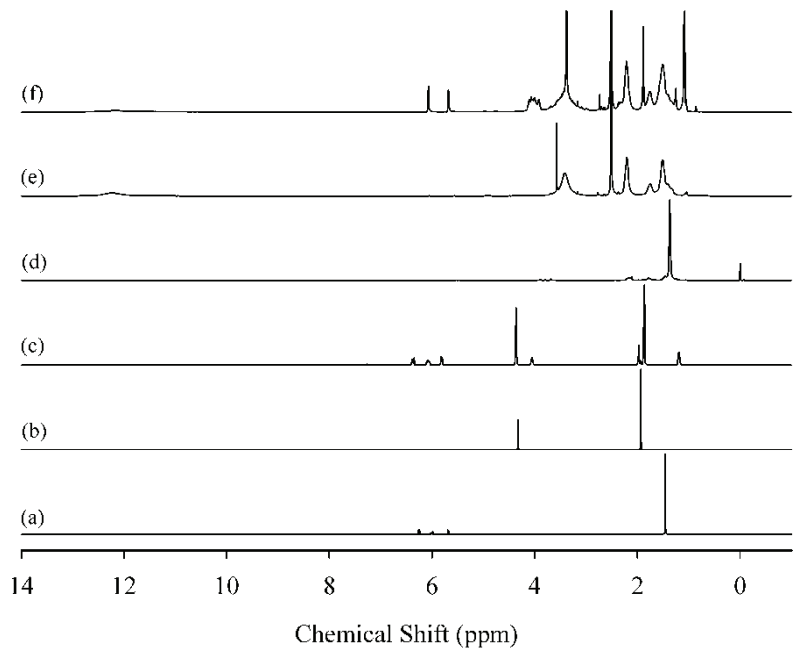

Figure 2. ${ }^{1} \mathrm{HNMR}$ spectra: (a) t-BA, (b) 4-arm BIBB, (c) BIEA, (d) poly(t-BA), (e) poly(AA) and (f) GM-tethered poly(AA).

BIEA, poly(t-BA), poly(AA) and GM-tethered poly(AA). The chemical shifts (ppm) were found and listed below: (a) t-BA: $1.50\left(-\mathrm{CH}_{3}, 9 \mathrm{H}\right), 5.68\left(=\mathrm{CH}_{2}, 1 \mathrm{H}\right), 6.00$ (= CHCO-, $1 \mathrm{H})$ and $6.27\left(=\mathrm{CH}_{2}, 1 \mathrm{H}\right)$; (b) 4-arm BIBB: $1.93\left(-\mathrm{C}\left(\mathrm{CH}_{3}\right)_{2}, 24 \mathrm{H}\right)$ and $4.32\left(\mathrm{CCH}_{2} \mathrm{O}, 8 \mathrm{H}\right)$; (c) BIEA: $1.86\left(-\mathrm{CH}_{3}, 6 \mathrm{H}\right), 4.36\left(-\mathrm{OCH}_{2} \mathrm{CH}_{2} \mathrm{O}-, 4 \mathrm{H}\right), 5.82\left(=\mathrm{CH}_{2}\right.$, $1 \mathrm{H}), 6.08$ (=CHCO-, $1 \mathrm{H})$ and $6.36\left(=\mathrm{CH}_{2}, 1 \mathrm{H}\right)$; (d) poly(t-BA): $1.38\left(-\mathrm{CH}_{3}\right), 1.78\left(-\mathrm{CH}_{2}^{-}\right)$and 2.15 (-CHCO-); (e) $\operatorname{poly}(\mathrm{AA}): 1.51\left(-\mathrm{CH}_{3}\right), 2.36\left(-\mathrm{CH}_{2}-\right), 3.37$ (-CHCO-) and $12.24(-\mathrm{COOH})$; and (f) GM-tethered poly(AA): $1.50\left(-\mathrm{CH}_{3}\right), 2.25\left(-\mathrm{CH}_{2}-\right), 3.25(\mathrm{OH}), 3.35$ (-CHCO-), 3.80-4.15 $\left(-\mathrm{OCH}_{2}-\right) 5.67\left(\mathrm{CH}_{2}=\right), 6.06\left(\mathrm{CH}_{2}=\right)$ and 12.22 $(-\mathrm{COOH})$.

\subsubsection{Polymerization Kinetics}

The ATRP polymerization kinetics of the star-hyperbranched poly(t-BA) was studied using ${ }^{1} \mathrm{HNMR}$. After the reaction was initiated, aliquots were retrieved from the reaction system at different time intervals, dissolved in $\mathrm{CDCl}_{3}$ and measured with ${ }^{1} \mathrm{HNMR}$ immediately. Figure 3 shows a kinetic plot of monomer to polymer conversion versus time and a semi-logarithmic plot of $\ln$ $\left([\mathrm{M}]_{0} /[\mathrm{M}]\right)$ versus time, where $[\mathrm{M}]_{0}=$ the initial concentration of the monomer and $[\mathrm{M}]=$ the monomer concentration at any time. The conversion was calculated by comparison of the peak integrations between 6.27 ( $\mathrm{HC}=$ C) and 1.2-1.6 ppm $\left(-\mathrm{CH}_{3}\right)$. The values of $\ln \left([\mathrm{M}]_{0} /[\mathrm{M}]\right)$ were obtained from ln $[1 /(1$-conversion\%)]. Two stages were found from the plot of $\ln \left([\mathrm{M}]_{0} /[\mathrm{M}]\right)$ versus time: a linear plot with 0.484 (slope) and $0.943\left(\mathrm{R}^{2}\right)$ within $3 \mathrm{~h}$ after the reaction was initiated and a deviated plot with a steeper slope after $3 \mathrm{~h}$. The conversion corresponding to the linear plot at $3 \mathrm{~h}$ was $78 \%$. The conversion reached $98.7 \%$ at $4 \mathrm{~h}$, indicating that the reaction was accelerated

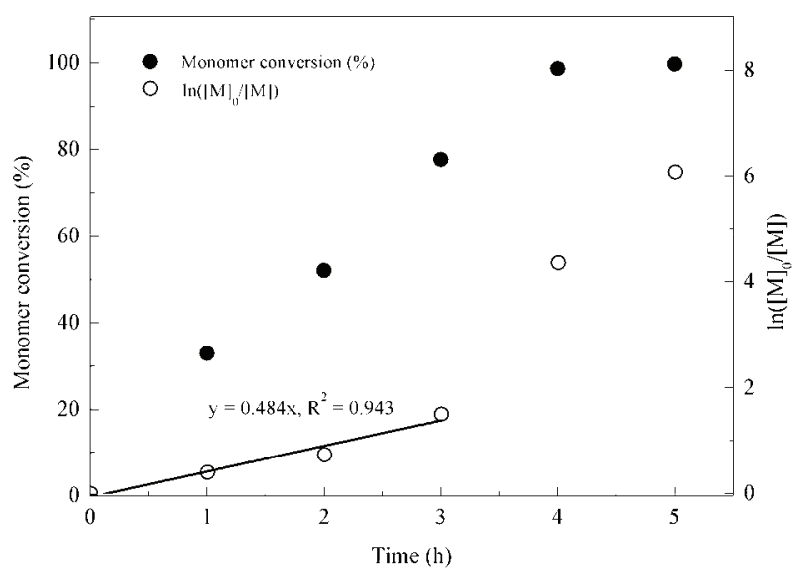

Figure 3. Conversion and kinetic plot of the star-hyperbranched poly(t-BA) derived from ${ }^{1} \mathrm{HNMR}$ spectra: 4-star/ $\mathrm{BIEA}=1: 4$ (by mole), (4-arm/BIEA)/t-BA = 1\% (by mole), $\mathrm{t}-\mathrm{BA} /$ dioxane $=50 / 50$ (by weight) and reaction temerature $=$ $120^{\circ} \mathrm{C}$.

after $3 \mathrm{~h}$.

\subsubsection{Effects of the Arm Number and Branching on MW, MWD and Viscosity}

The measured MW and MW distribution (MWD) of the synthesized star-hyperbranched poly(t-BA) and the viscosities of the corresponding polymer aqueous solutions are shown in Table 1. Increasing the arm number increased the MWD but decreased the solution viscosity. Increasing the branching increased the MWD but decreased the solution viscosity. In other words, both star arm number and branching increased the MW distribution of the synthesized poly(t-BA) but decreased the viscosity of the poly(AA) aqueous solution, indicating

Table 1. $M_{n}, M_{w}$, MWD and solution viscosity of the synthesized polymers ${ }^{1}$.

\begin{tabular}{ccccc}
\hline & $\mathrm{M}_{\mathrm{n}}$ (Dalton) & $\mathrm{M}_{\mathrm{w}}$ (Dalton) & MWD & Viscosity $\left(\mathrm{cP} \times 10^{-3}\right)^{4}$ \\
\hline \multicolumn{5}{c}{ Effect of arm number } \\
3 & 16630 & 55212 & 3.32 & 9.27 \\
4 & 17164 & 49089 & 2.86 & 6.11 \\
6 & 16725 & 91988 & 5.54 & 4.65 \\
& & Effect of branching & \\
$\mathrm{L}$ & 17164 & 49089 & 2.86 & 6.11 \\
$\mathrm{M}$ & 12274 & 46150 & 3.76 & 3.21 \\
$\mathrm{H}$ & 10575 & 44204 & 4.18 & 1.90 \\
\hline
\end{tabular}

${ }^{1} M_{n}$ (number average MW), $M_{w}$ (weight average MW) and MWD (MW distribution) were measured by GPC; ${ }^{2}$ The 3, 4 and 6 stands for star arm number, respectively; ${ }^{3} \mathrm{~L}, \mathrm{M}$ and $\mathrm{H}$ represents the BIEB used in low, medium and high level; ${ }^{4}$ Viscosity of the GM-tethered poly(AA) in water (polymer: water $=60: 40$, by weight) was determined at $23^{\circ} \mathrm{C}$. 
that more star arm number and more branching in the polymer can reduce the solution viscosity.

\subsection{Evaluation}

Table 2 shows the effects of the arm number and branching on CS, DTS, FS and compressive modulus of the experimental cements. There is a trend that increasing the arm number and branching decreased CS, DTS, FS and modulus, although most of the values in each category were not statistically different from one another. Table 3 shows the effects of the arm number and branching on KHN, FT, abrasion and attrition of the experimental cements. There is also a trend that increasing the arm number and branching decreased KHN, FT, abrasion resistance and attrition resistance, although some of the values in each category were not statistically different from one another. Figure 4 shows the effect of aging in water on CS, DTS and compressive modulus of the experimental cements. Significant increases were found in CS (253.6 to 320.2 MPa), DTS (60.8 to $67.9 \mathrm{MPa}$ ) and modulus (5.76 to $8.27 \mathrm{GPa}$ ) from 1 hour to 1 day. There were no statistically significant changes found among CS, DTS and modulus, although there is an increasing trend for all the strengths with the time.

Table 4 shows the mean values of CS, modulus, DTS, FS, FT, KHN, abrasion and attrition of the experimental cement (EXPGIC) versus commercial Fuji II LC cement. Apparently, EXPGIC exhibited significantly higher values than Fuji II LC in all the measured mechanical properties ( $<$ 0.05). Briefly, EXPGIC was 51\% in CS, 55\%

Table 2. Effects of arm number and branching on CS, DTS, FS and modulus of the cements ${ }^{1}$.

\begin{tabular}{|c|c|c|c|c|}
\hline & $\mathrm{CS}[\mathrm{MPa}]$ & DTS [ MPa] & FS [MPa] & Modulus [GPa $]^{2}$ \\
\hline \multicolumn{5}{|c|}{ Effect of arm number } \\
\hline 3 & $320.2(9.4)^{\mathrm{a}, 3}$ & $72.6(3.6)^{\mathrm{c}}$ & $114.2(14.1)^{\mathrm{f}}$ & $8.27(0.10)^{\mathrm{h}}$ \\
\hline 4 & $301.7(9.4)^{\mathrm{a}, \mathrm{b}}$ & $67.9(2.7)^{\mathrm{c}, \mathrm{d}}$ & $101.4(7.6)^{\mathrm{f}, \mathrm{g}}$ & $7.95(0.14)^{\mathrm{h}, \mathrm{i}}$ \\
\hline 6 & $286.3(8.9)^{\mathrm{b}}$ & $58.4(3.8)^{\mathrm{d}, \mathrm{e}}$ & $92.4(11.1)^{\mathrm{g}}$ & $7.56(0.22)^{\mathrm{i}}$ \\
\hline \multicolumn{5}{|c|}{ Effect of branching } \\
\hline L & $301.7(9.4)^{\mathrm{a}, \mathrm{b}}$ & $67.9(2.7)^{\mathrm{c}, \mathrm{d}}$ & $101.4(7.6)^{\mathrm{f}, \mathrm{g}}$ & $8.27(0.10)^{\mathrm{h}}$ \\
\hline M & $285.9(9.5)^{\mathrm{b}}$ & $58.8(3.6)^{\mathrm{d}, \mathrm{e}}$ & $88.4(9.1)^{\mathrm{g}}$ & $7.82(0.14)^{\mathrm{i}}$ \\
\hline $\mathrm{H}$ & $257.8(10)$ & $49.4(3.4)^{\mathrm{e}}$ & $89.1(15)^{g}$ & $7.49(0.27)^{\mathrm{i}}$ \\
\hline \multicolumn{5}{|c|}{$\begin{array}{l}{ }^{1} \text { Polymer }=\text { GM-tethered star-hyperbranched poly(AA), }(4-\text { star } / \mathrm{BIEA}) / \mathrm{t}-\mathrm{BA} \\
=1 \% \text { (by mole), GM grafting ratio }=50 \% \text { (by mole), P } / \mathrm{W} \text { ratio }=70 / 30(\mathrm{by} \\
\text { weight), P/L ratio }=2.7 \text { (by weight). }{ }^{2} \text { Modulus }=\text { compressive modulus } \\
{ }^{3} \text { Entries are mean values with standard deviations in parentheses and the } \\
\text { mean values with the same letter in each category were not significantly } \\
\text { different }(\mathrm{p}>0.05) \text {. Specimens were conditioned in distilled water at } 37^{\circ} \mathrm{C} \\
\text { for } 24 \mathrm{~h} \text { prior to testing. }\end{array}$} \\
\hline
\end{tabular}

Table 3. Effects of arm number and branching on KHN, FT, abrasion and attrition of the cements.

\begin{tabular}{ccccc}
\hline & KHN & FT $\left[\mathrm{MPa} \cdot \mathrm{m}^{0.5}\right]$ & $\begin{array}{c}\text { Abrasion } \\
{\left[\mathrm{nm} \cdot \text { cycle }^{-1}\right]}\end{array}$ & $\begin{array}{c}\text { Attrition } \\
{\left[\mathrm{nm} \cdot \text { cycle }^{-1}\right]}\end{array}$ \\
\hline & & Effect of arm number & \\
3 & $58.9(3.5)^{\mathrm{a}, 1}$ & $1.05(0.06)^{\mathrm{c}}$ & $0.41(0.12)$ & $0.71(0.06)^{\mathrm{e}}$ \\
4 & $58.5(0.6)^{\mathrm{a}}$ & $1.11(0.18)^{\mathrm{c}}$ & $0.26(0.05)^{\mathrm{d}}$ & $0.73(0.20)^{\mathrm{e}}$ \\
6 & $51.4(4.3)^{\mathrm{b}}$ & $1.06(0.13)^{\mathrm{c}}$ & $0.26(0.07)^{\mathrm{d}}$ & $1.29(0.32)^{\mathrm{f}}$ \\
& & Effect of branching & \\
$\mathrm{L}$ & $58.5(0.6)^{\mathrm{a}}$ & $1.11(0.18)^{\mathrm{c}}$ & $0.26(0.05)^{\mathrm{d}}$ & $0.73(0.20)^{\mathrm{e}}$ \\
$\mathrm{M}$ & $49.2(1.4)^{\mathrm{b}}$ & $1.11(0.22)^{\mathrm{c}}$ & $0.32(0.06)$ & $0.92(0.15)$ \\
$\mathrm{H}$ & $50.2(1.4)^{\mathrm{b}}$ & $1.08(0.13)^{\mathrm{c}}$ & $0.56(0.18)$ & $1.31(0.30)^{\mathrm{f}}$ \\
\hline
\end{tabular}

${ }^{1}$ Entries are mean values with standard deviations in parentheses and the mean values with the same letter in each category were not significantly different $(\mathrm{p}>0.05)$. Specimens were conditioned in distilled water at $37^{\circ} \mathrm{C}$ for $24 \mathrm{~h}$ prior to testing.

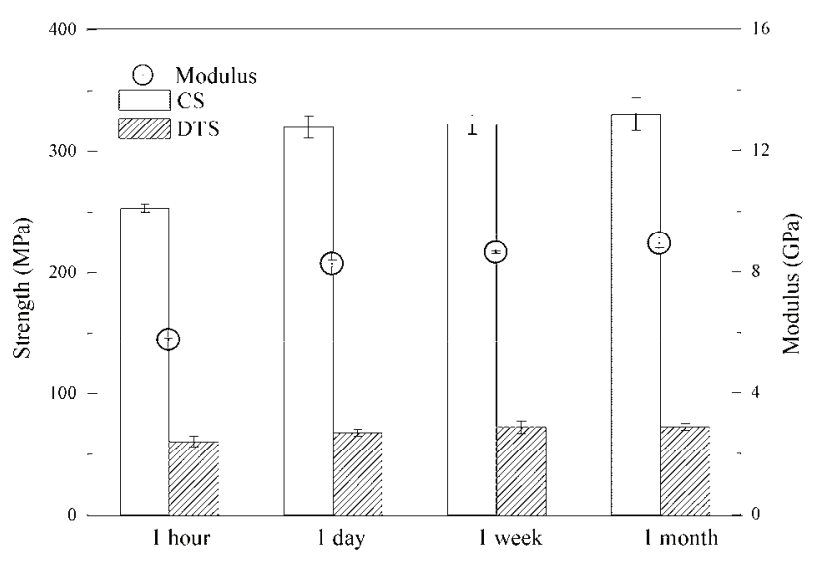

Figure 4. Strength change of the cement in the course of aging in water: polymer $=\mathrm{GM}$-tethered star-hyperbranched poly(AA), 4-arm/BIEA = 1:4 (by mole), $(4-$ star/BIEA) $/ \mathrm{t}-\mathrm{BA}=1 \%$ (by mole), GM grafting ratio $=50 \%$ (by mole), $\mathrm{P} / \mathrm{W}$ ratio $=70 / 30$ (by weight), $\mathrm{P} / \mathrm{L}$ ratio $=2.7$ (by weight). Specimens were conditioned in distilled water at $37^{\circ} \mathrm{C}$ prior to testing.

in compressive modulus, $118 \%$ in DTS, $82 \%$ in FS, $18 \%$ in FT and $85 \%$ in KHN higher than Fuji II LC. For wear test, EXPGIC was only $6.7 \%$ of abrasive and $10 \%$ of attritional wear depths of Fuji II LC in each wear cycle. Figures 5(a-f) illustrates representative regions of the fracture surfaces after the FS ((a) and (b)) and FT ((c) and (d)) tests, and the wear surfaces after the attrition ((e) and (f)) test, respectively, using a standardized magnification of $1500 \times$. The crack in the microstructure was caused by dehydration during preparation for SEM analysis [26]. In the case of Figures 5(a), (b), (c), (d), it is apparent that the fracture surfaces with EXPGIC ((b) and (d)) look denser, more integrated and more rugged 


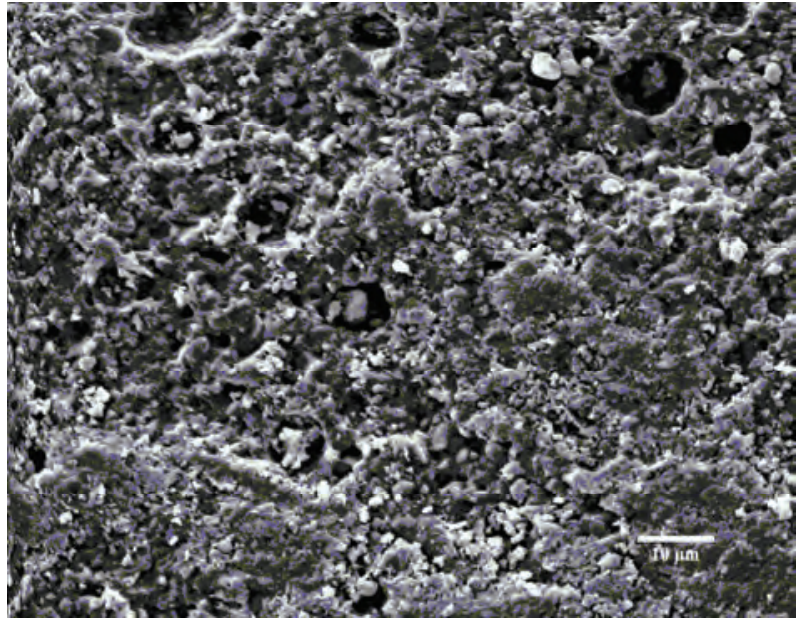

(a)

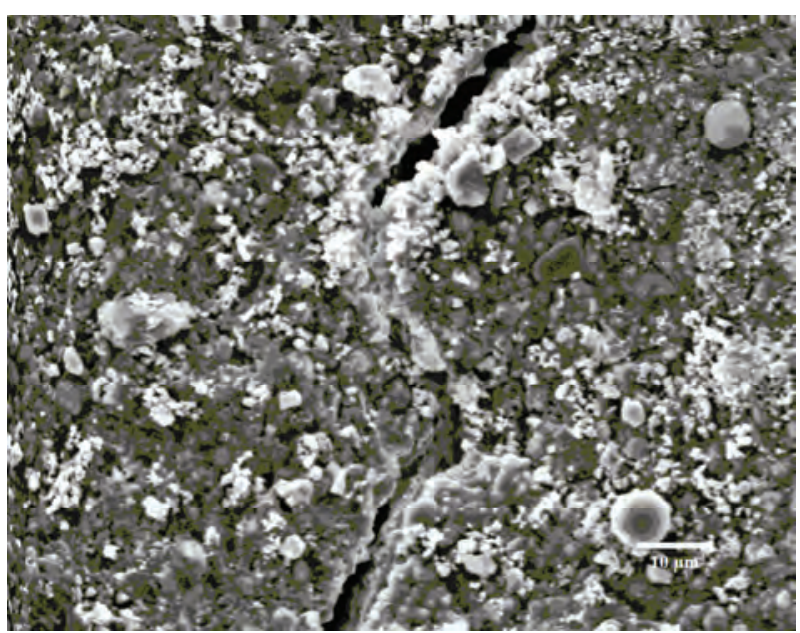

(c)

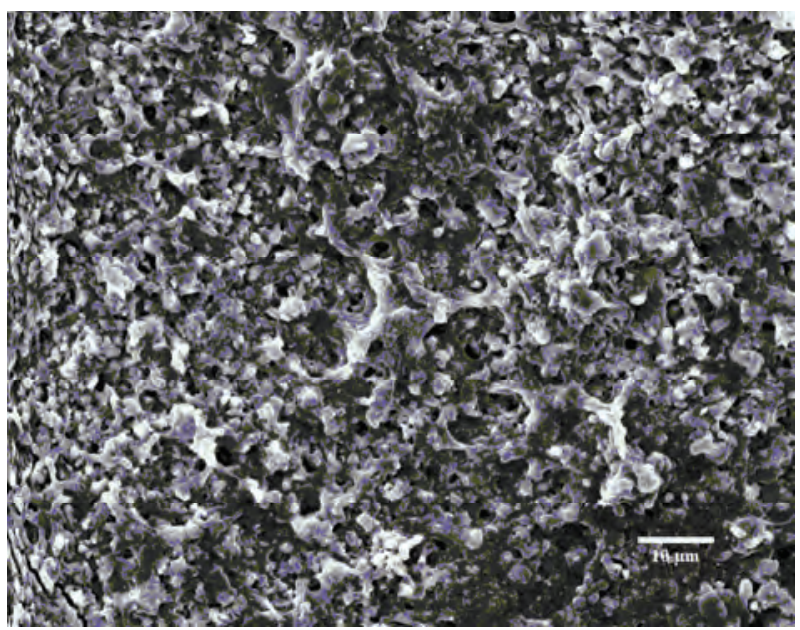

(e)

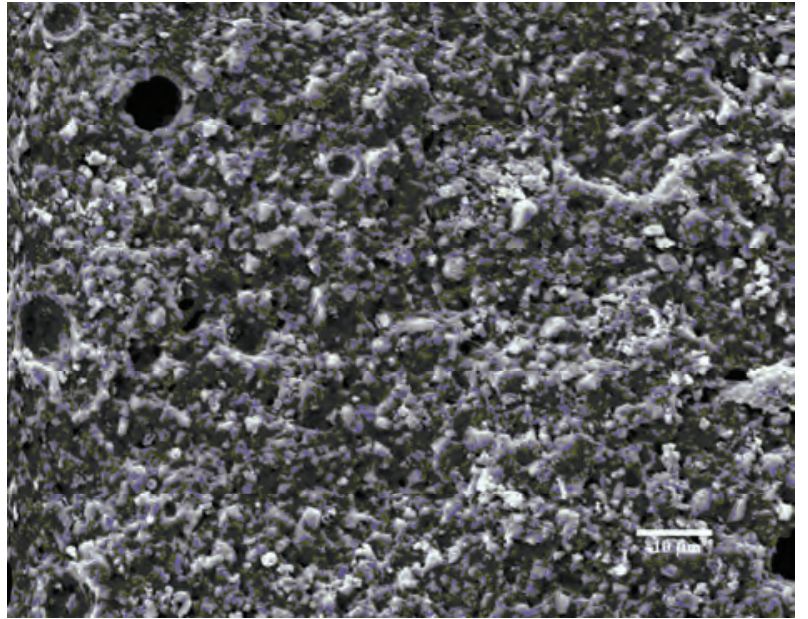

(b)

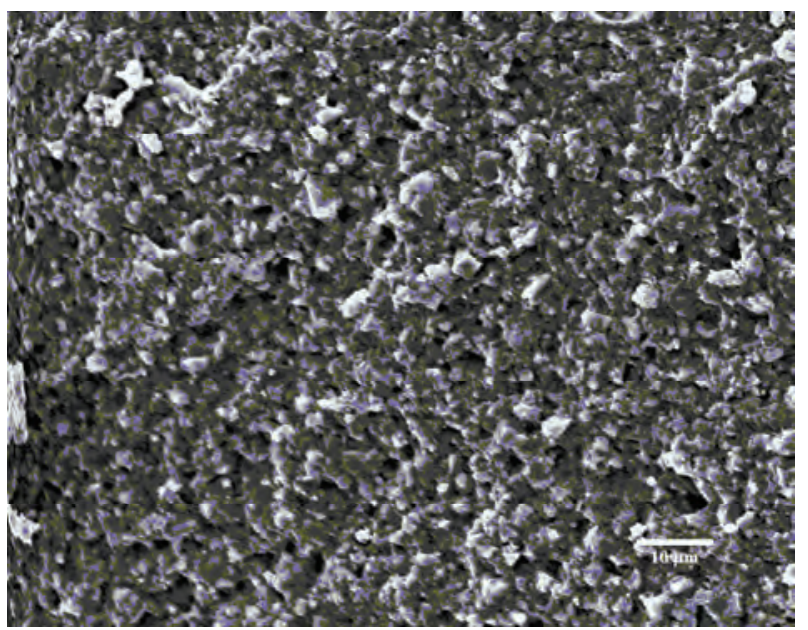

(d)

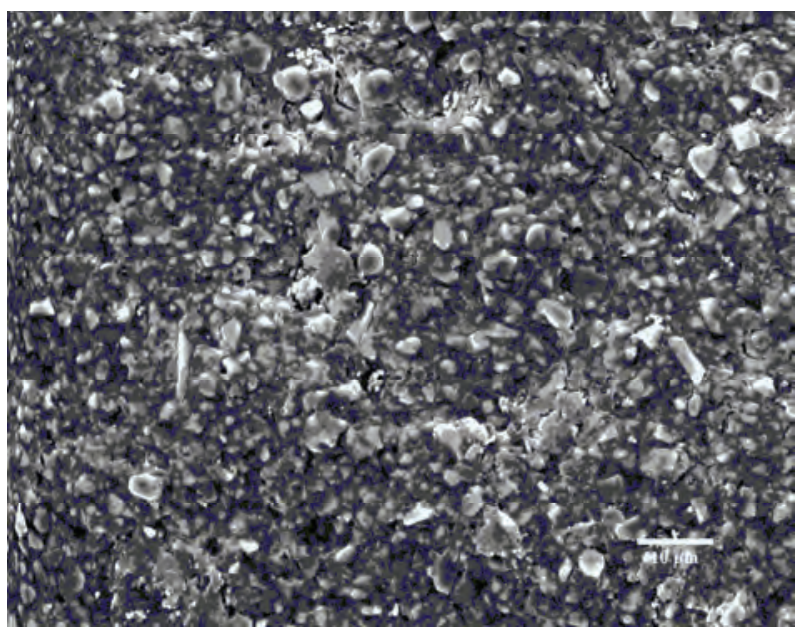

(f)

Figure 5. Fracture and wear surface photomicrographs at a magnification of 1500×: (a), (b) and (c) = fracture surface from FS, fracture surface from FT, attrition wear surface from wear tests, respectively, for Fuji II LC. (d), (e) and (f) = fracture surface from FS, fracture surface from FT, attrition wear surface from wear tests, respectively, for EXPGIC. Specimens were conditioned in distilled water at $37^{\circ} \mathrm{C}$ for $24 \mathrm{~h}$ prior to testing. 
Table 4. Mechanical property comparison between Fuji II LC and EXPGIC.

\begin{tabular}{lcc}
\hline \multicolumn{1}{c}{ Property } & Fuji II LC & EXPGIC $^{1}$ \\
\hline CS [MPa] & $212.7(15)^{2}$ & $320.2(9.4)$ \\
Modulus [GPa] & $5.33(0.09)$ & $8.27(0.1)$ \\
DTS [MPa] & $31.2(2.2)$ & $67.9(2.7)$ \\
FS [MPa] & $55.8(4.1)$ & $101.4(7.6)$ \\
FT [MPa·m $\left.{ }^{0.5}\right]$ & $0.94(0.01)$ & $1.11(0.18)$ \\
KHN & $31.7(1.0)$ & $58.5(0.6)$ \\
Abrasion [nm·cycle $\left.{ }^{-1}\right]$ & $3.90(0.81)$ & $0.26(0.05)$ \\
Attrition [nm·cycle $\left.{ }^{-1}\right]$ & $7.21(1.99)$ & $0.73(0.20)$ \\
\hline
\end{tabular}

${ }^{1}$ Polymer = GM-tethered star-hyperbranched poly(AA), 4-star/BIEA = 1:4 (by mole), (4-star/BIEA)/t-BA $=1 \%$ (by mole), GM grafting ratio $=50 \%$ (by mole), $\mathrm{P} / \mathrm{W}$ ratio $=70 / 30$ (by weight), $\mathrm{P} / \mathrm{L}$ ratio $=2.7$ (by weight); ${ }^{2}$ Entries are mean values with standard deviations in parentheses and all the mean values in each category were significantly different $(p<0.05)$. Specimens were conditioned in distilled water at $37^{\circ} \mathrm{C}$ for $24 \mathrm{~h}$ prior to testing.

than those with Fuji II LC ((a) and (c)). The rugged and highly integrated fragments suggest that the experimental cement was a tougher material than Fuji II LC. In contrast, loosely bonded fragments are observed in Fuji II LC. On the other hand, more big pores or voids are observed in Fuji II LC ((a) and (c)), indicating more air bubbles trapped during the cement preparation. For attrition ((e) and (f)), Fuji II LC (e) showed many crashed filler particles on a much rougher surface compared to EXPGIC (f). The wear surface of EXPGIC (f) looks much smoother than that for Fuji II LC.

\section{DISCUSSION}

\subsection{Synthesis and Characterization}

All the chemical shifts in Figure 2 confirmed the structures of the synthesized initiators and polymers. The characteristic chemical shifts at $3.25(\mathrm{OH}), 5.67\left(\mathrm{CH}_{2}=\right)$ and $6.06\left(\mathrm{CH}_{2}=\right)$ identified the difference between the star-hyperbranched poly(AA) and GM-tethered star-hyperbranched poly(AA).

Regarding the synthesis of the star-hyperbranched polymer via ATRP, it is known that ATRP reaction generally exhibits the first-order kinetics due to persistent radical effect [30]. As shown in Figure 3, $\ln \left([\mathrm{M}]_{0} /[\mathrm{M}]\right)$ increased linearly with time until the monomer conversion reached $78 \%$, indicating that the reaction followed the first-order kinetics at the early stage of polymerization. However, after $3 \mathrm{~h}$ the plot deviated more from linearity showing an accelerated fashion. The acceleration may be attributed to the reason that at a higher con- version the acrylate groups were located at the end of extended polymer chains, which may limit their mobility and thus reduce their reactivity with the propagating radicals. In addition, the solution viscosity was found to be significantly high at the later stage. These two reasons might lead to reduction of the termination constant and auto acceleration of the polymerization. As a result, the conversions were $98.7 \%$ at $4 \mathrm{~h}$ and $99.7 \%$ at $5 \mathrm{~h}$, respectively.

We have also studied the effects of arm number and branching on MW, WMD of the polymer and viscosity of the corresponding poly(AA) aqueous solution (see Table 1). From Table 1, the higher the star arm number, the lower the viscosity. This is logical because the 6 -arm star polymer is more like a sphere as compared to 3- and 4-arm star polymers. For the effect of the branching, we synthesized the star-hyperbranched polymers by changing the concentration of BIEA while keeping the starshape initiator constant. It was found that increasing BIEA or branching significantly decreased the viscosity of the polymer aqueous solution. It has been noticed that polymers with star, hyperbranched or dendritic shapes often demonstrate low solution or melt viscosity because these molecular structures behave similar to a solution of hard spheres and exhibit limited chain entanglements, which is beneficial to polymer processing $[19,20]$. In dental clinics, it is known that mixing a dental cement requires a workable solution viscosity for the polymer solution. Relatively low solution viscosity favors cement mixing clinically and reduces the probability of forming flaws or defects, thus enhancing the mechanical strength $[2,4,8]$. Therefore, without compromising the mechanical strengths a polymer solution with a low viscosity would be favorable to dental clinics. The results in Table 1 support that branching does decrease the viscosity.

The results in Tables 2 and 3 show that there is a trend that increasing either arm number or branching decreased the mechanical strengths, although the decrease was not statistically significant. This can be attributed to the fact that all the initiators we have used in this study are mainly composed of hydrocarbons and esters. None of them contain functional groups such as a carboxyl group for strength enhancement. The bulky hydrophobic initiator cores do not contribute any strength enhancement to the cement system. Therefore, the more the initiator in the system, the lower the mechanical strength is. Fortunately, these cores did not affect the strength significantly.

It is well known that GICs increase their strengths in water with time due to constant salt-bridge formations $[3,31]$. To demonstrate the unique nature of GICs in our new cement system, we measured the CS, DTS and compressive modulus values of the experimental cement 
after conditioning in water for $1 \mathrm{~h}, 1$ day, 1 week and 1 month (see Figure 4). The experimental cement showed a significant increase (26\% in CS, $12 \%$ in DTS and $44 \%$ in modulus) from 1 hour to 1 day. After that a slower increase was noticed.

Finally, we measured the mechanical properties of commercial Fuji II LC cement and compared them with those for the experimental cement. Table 4 shows all the measured mechanical properties of the optimal experimental cement (EXPGIC) vs. Fuji II LC. EXPGIC showed significantly higher mechanical strengths including CS, compressive modulus, DTS, FS, FT, KHN, abrasion and attrition than Fuji II LC. The higher mechanical strengths exhibited by EXPGIC can be attributed to the nature of this unique experimental cement system. As mentioned in the section of Materials and Methods, EXPGIC was composed of star-hyperbranched poly(AA) polymer, water and initiators. There were no any low MW comonomers in it. Essentially this is a monomer-free cement system. The polymer aqueous liquid contains highly concentrated GM-tethered starhyperbranched poly(AA), which not only provides a large quantity of carboxyl groups for salt-bridge formation but also a substantial amount of carbon-carbon double bond (methacrylate) for covalent crosslinks. In contrast, in addition to linear poly(AA) and water, Fuji II LC contains a substantial amount of HEMA (2-hydroxylethyl methacrylate, a low MW monomer) and other low MW methacrylate or dimethacrylate comonomers [13]. These low MW monomers and oligomers led to a lower strength. The fracture and wear surface photomicrographs from SEM (Figure 5) strongly supported the mechanical strength differences between the two cements.

\section{CONCLUSIONS}

This study developed a new glass-ionomer cement system composed of the newly synthesized star-hyperbranched poly(AA). The star-hyperbranched poly(AA) was synthesized via ATRP. The effects of arm number and branching on viscosity, CS, DTS and compressive modulus of the formed polymers and cements were evaluated. The results showed that both arm number and branching showed significant effects on viscosity and mechanical strengths. It was found that the more the arm number and branching that the polymer has, the lower the viscosity of the polymer aqueous solution as well as the mechanical strengths of the formed cement. Within the limitations of this study, the experimental cement formulated with the newly synthesized star-hyperbranched polymer exhibited significantly higher mechanical strengths than commercial Fuji II LC. The experimental cement was $51 \%$ in CS, $55 \%$ in compressive modulus, $118 \%$ in DTS, $82 \%$ in FS, $18 \%$ in FT and $85 \%$ in KHN higher than Fuji II LC. The experimental cement was only $6.7 \%$ of abrasive and $10 \%$ of attritional wear depths of Fuji II LC in each wear cycle. It appears that this novel experimental cement is a clinically attractive dental restorative and may potentially be used for high-wear and high-stress-bearing site restorations. Future studies will include bonding to tooth and in vitro biocompatibility tests.

\section{ACKNOWLEDGEMENTS}

This work was sponsored by NIH challenge grant (RC1) DE020614.

\section{REFERENCES}

[1] Smith, D.C. (1998) Development of glass-ionomer cement systems. Biomaterials, 19(6), 467-478.

[2] Wilson, A.D. and McLean, J.W. (1988) Glass-ionomer cements. Quintessence Publ Co., Chicago.

[3] Davidson, C.L. and Mjör, I.A. (1999) Advances in glass-ionomer cements. Quintessence Publ Co., Chicago.

[4] Wilson, A.D. (1990) Resin-modified glass-ionomer cement. The International Journal of Prosthodontics, 3(5), 425-429.

[5] Hotz, P., McLean, J.W., Sced, I. and Wilson, A.D. (1977) The bonding of glass-ionomer cements to metal and tooth substrates. British Dental Journal, 142(2), 41-47.

[6] Lacefield, W.R., Reindl, M.C. and Retief, D.H. (1985) Tensile bond strength of a glass-ionomer cement. The Journal of Prosthetic Dentistry, 53(2), 194-198.

[7] Forsten, L. (1977) Fluoride release from a glass-ionomer cement. Scandinavian Journal of Dental Research, 85, 503-504.

[8] Craig, R.G. (1997) Restorative Dental Materials. 10th Edition, Mosby-Year Book, Inc., St Louis.

[9] Nicholson, J.W., Braybrook, J.H. and Wasson, E.A. (1991) The biocompatibility of glass-poly(alkenoate) glass-ionomer cements: A review. Journal of Biomaterials Science (Polymer Edition), 2(4), 277-285.

[10] Hume, W.R. and Mount, G.J. (1988) In vitro studies on the potential for pulpal cytotoxicity of glass-ionomer cements. Journal of Dental Research, 67(6), 915-918.

[11] Guggenberger, R., May, R. and Stefan, K.P. (1998) New trends in glass-ionomer chemistry. Biomaterials, 19(6), 479-483.

[12] Mitra, S.B. (1991) Adhesion to dentin and physical properties of a light-cured glass-ionomer liner/base. Journal of Dental Research, 70(1), 72-74.

[13] Momoi, Y., Hirosaki, K., Kohno, A. and McCabe, J.F. (1995) Flexural properties of resin-modified "hybrid" glass-ionomers in comparison with conventional acidbase glass-ionomers. Dental Materials Journal, 14(2), 109-119.

[14] Xie, D., Culbertson, B.M. and Johnston, W.M. (1998) Formulations of light-curable glass-ionomer cements containing N-vinylpyrrolidone. Journal of Macromolecular Science, Part A Pure and Applied Chemistry, A35(10), 1631-1650.

[15] Xie, D., Wu, W., Puckett, A., Farmer, B. and Mays, J. 
(2004) Novel resin modified glass ionomer cements with improved flexural strength and ease of handling. European Polymer Journal, 40(2), 343-351.

[16] Kao, E.C., Culbertson, B.M. and Xie, D. (1996) Preparation of glass-ionomer cement using N-acryloyl-substituted amino acid monomers: Evaluation of physical properties. Dental Materials, 12(1), 44-51.

[17] Xie, D., Chung, I.-D., Wu, W., Lemons, J., Puckett, A. and Mays, J. (2004) An amino acid modified and nonHEMA containing glass-ionomer cement. Biomaterials, 25(10), 1825-1830.

[18] Xie, D., Culbertson, B.M. and Johnston, W.M. (1998) Improved flexural strength of $\mathrm{N}$-vinylpyrrolidone modified acrylic acid copolymers for glass-ionomers. Journal of Macromolecular Science, Part A Pure and Applied Chemistry, A35(10), 1615-1629.

[19] Bahadur, P. and Sastry, N.V. (2002) Principles of Polymer Science. CRC Press, Boca Raton.

[20] Huang, C.F., Lee, H.F., Kuo, S.W., Xu, H. and Chang, F.C. (2004) Star polymers via atom transfer radical polymerization from adamantine-based cores. Polymer, 45(7), 2261-2269.

[21] Xie, D., Park, J.G. and Zhao, J. (2007) Synthesis and preparation of novel 4-arm star-shape poly(carboxylic acid)s for improved light-cured glass-ionomer cements. Dental Materials, 23(4), 395-403.

[22] Xie, D., Yang, Y., Zhao, J., Park, J.G. and Zhang, J.T. (2007) A novel comonomer-free light-cured glass-ionomer cement for reduced cytotoxicity and enhanced mechanical strength. Dental Materials, 23(8), 994-1003.

[23] Ibrahim, K., Lofgren, B. and Seppala, J. (2003) Synthesis of tertiary-butyl acrylate polymers and preparation of diblock copolymers using atom transfer radical polymerization. European Polymer Journal, 39(10), 2005-
2010.

[24] Davis, K.A., Charleux, B. and Matyjaszewski, K. (2000) Preparation of block copolymers of polystyrene and poly(t-butyl acrylate) of various molecular weights and architectures by atom transfer radical polymerization. Journal of Polymer Science Part A: Polymer Chemistry, 38(12), 2274-2283.

[25] Johnson, W.W., Dhuru, V.B. and Brantley, W.A. (1993) Composite microfiller content and its effect on fracture toughness and diametral tensile strength. Dental Materials, 9(2), 95-98.

[26] Xie, D., Brantley, W.A., Culbertson, B.M. and Wang, G. (2000) Mechanical properties and microstructures of glass-ionomer cements. Dental Materials, 16(2), 129138.

[27] Dowling, A.H. and Fleming, G.J.P. (2007) The impact of montmorillonite clay addition on the in vitro wear resistance of a glass-ionomer restorative. Journal of Dentistry, 35(4), 309-317.

[28] Condon, J.R. and Ferracane, J.L. (1996) Evaluation of composite wear with a new multi-mode oral wear simulator. Dental Materials, 12(4), 218-226.

[29] Turssi, C.P., Ferracane, J.L. and Vogel, K. (2005) Filler features and their effects on wear and degree of conversion of particulate dental resin composites. Biomaterials, 26(24), 4932-4937.

[30] Matyjaszewski, K. and Xia, J. (2001) Atom transfer radical polymerization. Chemical Reviews, 101(9), 29212990.

[31] Cattani-Lorente, M.A., Godin, C. and Meyer, J.M. (1994) Mechanical behavior of glass ionomer cements affected by long-term storage in water. Dental Materials, 10(1), 37-44. 


\section{LIST OF THE ABBREVIATIONS}

\section{COMMONLY SHOWN IN THE PAPER:}

GIC-glass-ionomer cement;

CS-compressive strength;

DTS-diametral tensile strength;

FS-flexural strength;

KHN-Knoop hardness number;

FT-fracture toughness;

ATRP-atom-transfer radical polymerization;

MW-molecular weight;

MWD = molecular weight distribution;

EXPGIC-experimental glass-ionomer cement;

AA-acrylic acid;

GM-glycidyl methacrylate;

BIEA-2-(2-bromoisobutyryloxy) ethyl acrylate;

BIBB-2-bromoisobutyryl bromide;

t-BA-tert-butyl acrylate. 